\title{
Olmesartan for non-alcoholic steatohepatitis complicated with hypertension: An open-label study*
}

\author{
Hisashi Hidaka ${ }^{1 \#,}$ Makoto Ohbu $^{2}$, Yasuhiro Matsumoto ${ }^{1}$, Tsutomu Minamino ${ }^{1}$, Juichi Takada ${ }^{1}$, \\ Yoshiaki Tanaka ${ }^{1}$, Yusuke Okuwaki ${ }^{1}$, Masaaki Watanabe ${ }^{1}$, Takahide Nakazawa ${ }^{1}$, Akitaka Shibuya ${ }^{1}$, \\ Wasaburo Koizumi ${ }^{1}$ \\ ${ }^{1}$ Department of Gastroenterology, Kitasato University School of Medicine, Sagamihara, Kanagawa, Japan \\ ${ }^{2}$ Department of Pathology, Kitasato University School of Allied Health Science, Sagamihara, Kanagawa, Japan \\ Email: "hisashi7@kitasato-u.ac.jp
}

Received 2 March 2013; revised 3 April 2013; accepted 5 May 2013

Copyright (C) 2013 Hisashi Hidaka et al. This is an open access article distributed under the Creative Commons Attribution License, which permits unrestricted use, distribution, and reproduction in any medium, provided the original work is properly cited.

\begin{abstract}
Aim: We evaluated the long-term effects of olmesartan, an angiotensin type 1 receptor blocker, in patients with non-alcoholic steatohepatitis (NASH) complicated with hypertension. Methods: All patients were given a standard calorie diet and exercise counseling more than 3 months before the treatment. Seven patients with NASH received olmesartan treatment for 1 year. Liver biopsy, clinical parameters and blood markers of hepatic fibrosis, including serum hyaluronic acid, type IV collagen, and procollagen III Nterminal propeptide levels, were also examined at the beginning and the end of the study. Results: The median dose of the final administration was $20 \mathrm{mg}$ (range, 10 - $40 \mathrm{mg}$ ). Olmesartan reduced MAP by $-11.3 \pm 13.0 \%(P=0.046)$ after 1 year. In the laboratory data, serum AST, ALT, and ferritin significantly decreased after a year of administration (AST, $62 \pm$ 24 vs. $39 \pm 20$ IU/L, $P=0.018$; ALT, $106 \pm 79$ vs. $55 \pm$ 35 IU/L, $P=0.043$; ferritin, $323.8 \pm 252.8$ vs. $202.3 \pm$ $194.1 \mathrm{ng} / \mathrm{ml}, P=0.028)$. Furthermore, fasting glucose significantly decreased. However, transforming growth factor-beta1, the serum concentration of the fibrosis markers, and all histological features were unchanged at the end of the study. No side effects of the treatment were noted at any time during the study. Conclusion: Olmesartan significantly reduced blood pressure, fasting glucose, aminotransferase, and serum ferritin but could not suppress the hepatic fibrosis markers or histological features after 1 year. There-
\end{abstract}

\footnotetext{
"The authors who have taken part in this study declared that they do not have anything to disclose regarding funding or conflict of interest with respect to this manuscript. The trial described in this work has been registered under trial number: UMIN000005409.

"Corresponding author.
}

fore, olmesartan is advisable only for its anti-inflammatory effect in patients with NASH-complicated hypertension.

Keywords: Angiotensin Type 1 Receptor Blocker; Hepatic Fibrosis; TGF-Beta 1

\section{INTRODUCTION}

Non-alcoholic fatty liver disease (NAFLD) is now the most frequent cause of chronic liver disease in many countries, and is histologically categorized into non-alcoholic fatty liver and nonalcoholic steatohepatitis (NASH) [1]. NASH can progress to cirrhosis and increase risk of hepatocellular carcinoma [2], and is associated with metabolic risk factors, such as obesity, diabetes mellitus, dyslipidemia, and hypertension [1,2]. The histological findings of NASH are characterized by steatosis, lobular inflammation, and fibrosis of the liver. However, an effective drug therapy for NASH has not yet been established [1].

Angiotensin II (Ang II), the main peptide of the rennin-angiotensin system (RAS) regulates cell growth, inflammation, fibrosis, and contributes to the progression of injury of various organs through angiotensin type 1 (AT1) receptors [3]. The profibrogenic effect of Ang II is associated with an increased concentration of transforming growth factor-betal (TGF-beta1) [4]. Induction of TGF-beta1 by Ang II stimulates synthesis of matrix proteins, inhibits matrix degradation, and enhances expression of integration that facilitates matrix assembly [5]. In the liver, the RAS is also involved in chronic inflammation and fibrosis. TGF-betal produced from Kupffer cells and infiltrating inflammatory cells activates hepatic stellate cells (HSCs) [6]. AT1 receptors are expressed on 
activated HSCs, and Ang II enhanced hepatic fibrosis through the production of TGF-betal in animal models [7]. Moreover, the previous studies have suggested that RAS inhibitors (losartan) might diminish fibrosis progression in patients with NASH [8].

Olmesartan medoxomil (olmesartan) is a comparatively new angiotensin receptor blocker (ARB) [9], and the active metabolite olmesartan has been demonstrated to be a potent, selective ARB for hypertension [10]. Moreover, in the preclinical study, olmesartan significantly attenuated increases in aspartate aminotransferase, activation of hepatic stellate cells, oxidative stress, expression of TGF-beta1, expression of collagen genes, and liver fibrosis [11]. Therefore, this agent may possibly elicit a better outcome for patients with NASH-complicated hypertension than other ARBs. The aim of the present study was to investigate the effect of olmesartan in patients with NASH complicated hypertension.

\section{METHODS}

\subsection{Patients}

A prospective open-label study was conducted in patients with biopsy-proven NASH, and who were recruited from the Kitasato University East Hospital, Sagamihara, Kanagawa, Japan. Before the study, all patients were given a standard calorie diet $(25-30 \mathrm{kcal} / \mathrm{kg}$ per day, carbohydrate $50 \%$ - 60\%, fat $20 \%$ - 30\%, protein $15 \%$ - 20\%) and exercise counseling for longer than 3 months. Consecutive NASH patients with hypertension received olmesartan treatment for 1 year. The final protocol was approved by the Ethics Committee of the Kitasato University School of Medicine, Sagamihara, Kanagawa, Japan (C-Ethics Committee, ID 08-432). The study was conducted following the principles of the Declaration of Helsinki. After obtaining written, informed consent, patients with the following inclusion criteria, and none of the exclusion criteria, were enrolled in the study from September 2008 to August 2011. The trial described in this work has been registered under the following trial number: www.umin.ac.jp/ctr/index.htm, UMIN 000005409.

The inclusion criteria were: 1) age between 18 and 75 years; 2) proven NASH with a diagnosis based on histology; 3) systolic blood pressure $\geq 140 \mathrm{mmHg}$ and/or diastolic blood pressure $\geq 90 \mathrm{mmHg}$; 4) Child-Pugh score $\leq 9$. And the exclusion criteria were: 1 ) history of hepatic disease, such as hepatitis B (serum positive for hepatitis B surface antigen) or hepatitis C, autoimmune hepatitis, primary biliary cirrhosis, sclerosing cholangitis, hemochromatosis, alpha1-antitrypsin deficiency, Wilson's disease; 2) current or past consumption of more than 20 g of alcohol daily; 3) ARB intolerance; 4) current or past treatment with $\mathrm{ARB}$, angiotensin-converting enzyme in- hibitors, thiazolidinediones, metformin, or statins; 5) hypertrophic cardiomyopathy or renal arterial stenosis; 6) serum creatinine $>1.6 \mathrm{mg} / \mathrm{dL} ; 7$ ) hyperkalemia defined as plasma potassium $>5.5 \mathrm{mEq} / \mathrm{L}$; 8) current critical esophagogastric varices; and 9) hepatocellular carcinoma or portal venous thrombosis. Treatment with furosemide was allowed in order to check for ascites throughout the study period if necessary.

\subsection{Study Protocol}

Patients were given olmesartan $\left(\right.$ Olmetec $^{\circledR}$; Daiichi-Sankyo Co., Ltd., Tokyo, Japan) at a starting dose of $10 \mathrm{mg}$ daily, in the morning, which was increased stepwise according to the manufacture's recommendations (e.g., from $10 \mathrm{mg}$ to $20 \mathrm{mg}$ then to $40 \mathrm{mg}$ ) at 2-week intervals up to the maximum dose of $40 \mathrm{mg}$ daily, if tolerated, as long as the systolic blood pressure did not decrease below $90 \mathrm{mmHg}$, (because $40 \mathrm{mg}$ daily is recommended as the maximum daily dose in the general patient population) [9]. Once the maintenance dose was reached, treatment was maintained for 48 weeks.

All patients underwent bi-weekly clinical check-ups until the end of the study. Serum biochemistry and blood pressure were monitored at the beginning of the study regimen and every 4 weeks thereafter. Liver biopsy, abdominal ultrasonography, clinical parameters, and blood markers for hepatic fibrosis, including serum hyaluronic acid (HA), type IV collagen, and procollagen III N-terminal propeptide (PIIINP) levels, were also examined at the beginning and the end of the study. The occurrence of severe orthostatic symptoms concomitant with severe arterial hypotension, defined as mean arterial pressure (MAP) $<60 \mathrm{mmHg}$ during the treatment, was considered a severe side effect that required withdrawal of the medication.

\subsection{Assessment of liver Histology}

All entry and end-of-study liver biopsies were reviewed and scored by a dedicated liver pathologist blinded to the clinical and biochemical data. The NAFLD Activity Score (NAS) is the unweighted sum of steatosis, lobular inflammation, and hepatocellular ballooning scores [12]. Fibrosis at the end of the trial was staged 0 to 4 (0: absent; 1: perisinusoidal or portal/periportal only; 2: perisinusoidal and portal/periportal; 3: bridging fibrosis; 4: cirrhosis) [13].

\subsection{Assessment of Insulin Resistance}

Insulin resistance was calculated using the modified Homeostatic Model of Assessment of Insulin Resistance (HOMA-IR) according to the following formula: HOMA$\mathrm{IR}=$ fasting insulin $(\mu \mathrm{U} / \mathrm{ml}) \times$ plasma glucose $(\mathrm{mg} / \mathrm{dl}) /$ 
405. The HOMA-IR was originally reported by Matthews et al. and has since been modified [14].

\subsection{Sample Size Calculation}

We set up the primary endpoint as the significant reduction of TGF-betal after 1 year because Yokohama et al. previously reported an estimated the reduction rate of more than $40 \%$ in the ARB group [8]. At least a $10 \%$ failure rate in the group was previously estimated. On the basis of this $40 \%$ difference in 1 year, the minimum sample size of 7 per group would provide $80 \%$ power with a type 1 error of 0.05 for significance.

\subsection{Statistical Analysis}

All the data are presented as mean \pm SD. The paired or non-paired Student $t$-test was used to assess the significance of the comparison of the normally distributed data, and the Mann-Whitney $U$ or the Wilcoxon test was used for the non-normally distributed data. All $P$ values were two-tailed. A value of $P<0.05$ was considered to indicate a statistically significant difference. Statistical analysis was performed with the statistical package SPSS Base 17.0 J for Windows (Microsoft).

\section{RESULTS}

\subsection{Patients and Baseline Characteristics}

Between September 2008 and August 2011, a total of 11 patients with NASH were referred for the study after screening (Figure 1). In all, 4 patients were excluded from the study. Three patients had been already treated with insulin sensitizing agents, and 1 patient was confirmed as having critical esophagogastric varices. Therefore, the remaining 7 patients were included the study $(\mathrm{N}$ $=7$ ). The main characteristics of these patients are summarized in Tables 1 and 2. Median BMI before treatment was $26.5(23.5-32.5) \mathrm{kg} / \mathrm{m}^{2}$, and no significant changes were seen between before or after treatment. The therapy was well accepted and tolerated. The median dose of the final administration was $20 \mathrm{mg}$ (range, 10 $40 \mathrm{mg}$ ); 3 patients were treated with a maintenance dose of $40 \mathrm{mg}, 3$ patients with $20 \mathrm{mg}$, and 1 patient with 10 $\mathrm{mg}$.

\subsection{Hemodynamic Changes and Laboratory Data}

The changes in laboratory data at the baseline and after a year of treatment with olmesartan are shown in Table 2. Olmesartan reduced MAP by $-11.3 \% \pm 13.0 \%(P=$ 0.046) after 1 year. In the laboratory data, serum AST, ALT, and ferritin significantly decreased after a year of administration (AST, $62 \pm 24$ vs. $39 \pm 20 \mathrm{IU} / \mathrm{L}, P=$ 0.018 ; ALT, $106 \pm 79$ vs. $55 \pm 35$ IU/L, $P=0.043$; ferritin, $323.8 \pm 252.8$ vs. $202.3 \pm 194.1 \mathrm{ng} / \mathrm{ml}, P=0.028$ ). Furthermore, fasting glucose significantly decreased $(126 \pm 35$ vs. $115 \pm 34 \mathrm{mg} / \mathrm{dl}, P=0.043)$, however, HbA1c, IRI (immunoreactive insulin), and HOMA-IR did not change significantly after treatment.

\subsection{TGF-beta1 and Blood Markers of Hepatic Fibrosis}

TGF-betal did not decrease significantly at the end of the present study $(8.2 \pm 11.5$ vs. $4.1 \pm 2.4 \mathrm{ng} / \mathrm{ml}, P=$ $0.735)$. Furthermore, the serum concentration of the fibrosis markers, PIIINP, type IV collagen, and HA, were unchanged at the end of the study (Table 2).

\subsection{Histological Examination}

Follow-up histological examinations were available for all patients. Table 3 shows the histological changes before and after treatment. There were no significant changes in any of the histological features. Briefly, in the NAS after treatment, 4 of 7 (57.1\%) patients showed improvement or no change, and 3 of $7(42.9 \%)$ patients became worse. In the fibrosis score after treatment, 5 of $7(71.4 \%)$ patients showed improvement or no change, and 2 of 7 (28.6\%) patients became worse (Table 3).

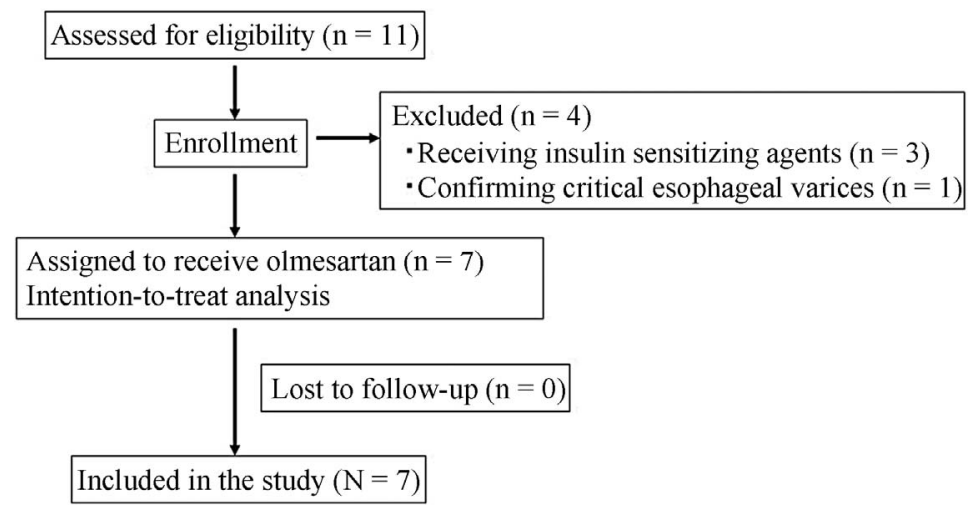

Figure 1. Flow diagram of study recruitment and follow-up. 
Table 1. Patient baseline clinical characteristics.

\begin{tabular}{cc}
\hline Variable & $\mathrm{N}=7$ \\
\hline Age (years) & $49.1 \pm 16.5$ \\
Sex $(\mathrm{M} / \mathrm{F})$ & $5 / 2$ \\
BMI $\left(\mathrm{kg} / \mathrm{m}^{2}\right)$ & \\
$<25$ & $1(14 \%)$ \\
$25-29$ & $4(57 \%)$ \\
$\geq 30$ & $2(29 \%)$ \\
Hypertension & $7(100 \%)$ \\
Hypercholesterolemia & $2(29 \%)$ \\
Hyperglyceridemia & $2(29 \%)$ \\
\hline
\end{tabular}

BMI, body mass index.

\subsection{Tolerability and Side Effects of Olmesartan Treatment}

All patients successfully completed the entire protocol. Serum K significantly increased after treatment $(4.0 \pm$ 0.2 vs. $4.2 \pm 0.3 \mathrm{mEq} / \mathrm{L}, P=0.048$ ). However, serum $\mathrm{K}$ level of all the patients had been within normal limits. No treatment side effects were seen at any time during the study.

\section{DISCUSSION}

We demonstrated that 1-year administration of olmesartan significantly reduced serum aminotransferase and ferritin. Furthermore, mean blood pressure (MBP) was

Table 2. Changes of clinical and laboratory data.

\begin{tabular}{|c|c|c|c|}
\hline Variable & Before treatment & After treatment & $P$-value \\
\hline No. of patients & 7 & 7 & \\
\hline Mean blood pressure $(\mathrm{mmHg})$ & $103 \pm 12$ & $92 \pm 14$ & 0.046 \\
\hline $\operatorname{BMI}\left(\mathrm{kg} / \mathrm{m}^{2}\right)$ & $27.9 \pm 3.5$ & $27.7 \pm 3.4$ & 0.686 \\
\hline Platelet $\left(\times 10^{4} / \mu \mathrm{L}\right)$ & $17.3 \pm 6.3$ & $18.3 \pm 4.9$ & 0.799 \\
\hline Total Bilirubin (mg/dl) & $1.0 \pm 0.4$ & $0.7 \pm 0.2$ & 0.093 \\
\hline AST (IU/l) & $62 \pm 24$ & $39 \pm 20$ & 0.018 \\
\hline ALT (IU/l) & $106 \pm 79$ & $55 \pm 35$ & 0.043 \\
\hline $\operatorname{gammaGTP}(\mathrm{IU} / \mathrm{l})$ & $74 \pm 41$ & $50 \pm 17$ & 0.091 \\
\hline Serum K (mE/L) & $4.0 \pm 0.2$ & $4.2 \pm 0.3$ & 0.048 \\
\hline Creatinine $(\mathrm{mg} / \mathrm{dL})$ & $0.72 \pm 0.17$ & $0.77 \pm 0.22$ & 0.141 \\
\hline LDL cholesterol (mg/dL) & $123 \pm 28$ & $151 \pm 33$ & 0.665 \\
\hline HDL cholesterol (mg/dL) & $51 \pm 11$ & $46 \pm 7$ & 0.655 \\
\hline Fasting glucose (mg/dL) & $126 \pm 35$ & $115 \pm 34$ & 0.043 \\
\hline HbA1c (\%) & $5.9 \pm 1.2$ & $5.8 \pm 1.3$ & 0.496 \\
\hline $\mathrm{IRI}(\mu \mathrm{U} / \mathrm{mL})$ & $13.8 \pm 6.4$ & $12.8 \pm 9.1$ & 0.500 \\
\hline HOMA-IR & $4.2 \pm 2.0$ & $2.8 \pm 1.8$ & 0.138 \\
\hline TGF-beta1 & $8.2 \pm 11.5$ & $4.1 \pm 2.4$ & 0.735 \\
\hline Type IV collagen (ng/mL) & $4.6 \pm 1.2$ & $4.2 \pm 1.1$ & 0.078 \\
\hline Type III procollagen-N-peptide (U/mL) & $0.62 \pm 0.13$ & $0.55 \pm 0.07$ & 0.225 \\
\hline Hyaluronic acid (ng/mL) & $26.2 \pm 26.4$ & $30.6 \pm 28.8$ & 0.174 \\
\hline Ferritin (ng/mL) & $323.8 \pm 252.8$ & $202.3 \pm 194.1$ & 0.028 \\
\hline Adiponectin $(\mu \mathrm{g} / \mathrm{mL})$ & $10.9 \pm 9.9$ & $10.4 \pm 9.8$ & 0.715 \\
\hline
\end{tabular}

ALT, alanine aminotransferase; AST, alanine aminotransferase; BMI, body mass index, gamma GTP, gamma-glutamyl transpeptidase; HDL, high-density lipoprotein; HOMA-IR, homeostasis model assessment of insulin resistance; IRI, immunoreactive insulin; LDL, low-density lipoprotein; TGF-beta1, transforming growth factor-beta1.

Table 3. Histological changes in patients with nonalcoholic seatohepatitis treated with.

\begin{tabular}{ccccccccccc}
\hline & \multicolumn{2}{c}{ NAFLD activity } & \multicolumn{2}{c}{ Steatosis } & \multicolumn{2}{c}{ Lobular inflammation } & \multicolumn{2}{c}{ Ballooning } & \multicolumn{2}{c}{ Fibrosis } \\
\hline & \multicolumn{2}{c}{ Score } & \multicolumn{2}{c}{ Score } & \multicolumn{2}{c}{ Score } & \multicolumn{2}{c}{ Score } & \multicolumn{2}{c}{ Score } \\
\hline Patient & Before & After & Before & After & Before & After & Before & After & Before & After \\
\hline 1 & 5 & 6 & 1 & 3 & 2 & 1 & 2 & 2 & 2 & 1 \\
2 & 4 & 4 & 1 & 1 & 2 & 2 & 1 & 1 & 1 & 1 \\
3 & 6 & 7 & 2 & 3 & 2 & 2 & 2 & 2 & 2 & 3 \\
4 & 5 & 4 & 1 & 1 & 2 & 1 & 2 & 2 & 4 & 3 \\
5 & 7 & 7 & 3 & 3 & 2 & 2 & 2 & 2 & 1 & 2 \\
6 & 4 & 5 & 1 & 1 & 1 & 2 & 2 & 2 & 3 & 3 \\
7 & 6 & 5 & 2 & 1 & 2 & 1 & 2 & 2 & 1 & 1 \\
& $5.3 \pm 1.1$ & $5.4 \pm 1.3$ & $1.6 \pm 0.8$ & $1.9 \pm 1.1$ & $1.9 \pm 0.4$ & $1.6 \pm 0.5$ & $1.9 \pm 0.4$ & $1.9 \pm 0.4$ & $2.0 \pm 1.6$ & $2.0 \pm 1.0$ \\
\hline
\end{tabular}


significantly reduced after 1 year. These results suggested that olmesartan may have an anti-inflammatory effect, at least in patients with NASH complicated with hypertension. It has been recognized that $40 \%$ of Japanese patients with NASH are complicated with hypertension [15]. Therefore, olmesartan should be considered for these NASH patients.

To our knowledge, there is only one prospective human study of ARB alone for NASH patients complicated with hypertension [8]. In that study, 7 patients with NASH-complicated hypertension were treated with losar$\tan (50 \mathrm{mg} /$ day $)$ for 48 weeks, which resulted in a significant decrease in plasma TGF-betal and serum ferritin concentration concurrently with an improvement in serum aminotransferase levels [8]. Histological assessment showed improvement of hepatic necroinflammation in 5 patients, reduction of hepatic fibrosis in 4 , and disappearance of iron deposition in 2 patients. These results were a little different from those in the present study. We could not clearly explain why olmesartan did not show an antifibrotic effect in the present study, because $20 \mathrm{mg}$ of olmesartan, which is the median dose of the final administration, was more effective for hypertensive patients than $50 \mathrm{mg}$ of losartan in a multicenter, randomized, double-blind trial [9].

It is necessary to keep in mind that there were no treatment side effects at any time during the present study. There are several treatments for NASH, and exercise alone in adults with NASH may reduce hepatic steatosis [1]. In addition to obesity, NASH is sometimes associated with type 2 diabetic mellitus, hyperlipidemia and hypertension. Insulin sensitizing agents (metformin and thiazolidinediones) for NASH complicated with type 2 diabetic mellitus have been thought to be effective. However, recent meta-analyses concluded that $6-12$ months of metformin plus lifestyle intervention did not improve aminotransferase or liver histology compared with lifestyle intervention alone $[1,4]$. Pioglitazone (thiazolidinediones) is only recommended for NASH patients with type 2 diabetic mellitus [1]. However, it is limited by side effects, such as weight gain, painful swollen legs, osteopenia, and cardiovascular disease [1620]. Consequently, we have not obtained any confirmed and safe drugs for NASH yet.

It is necessary to consider the efficacy of other ARBs alone or the combination therapy with other drugs for NASH. Recently, it was designed to compare the three regimens for the patients with NASH: rosiglitazone alone, rosiglitazone plus metformin, and rosiglitazone plus losartan $(50 \mathrm{mg})$ [16]. In this study, 48 weeks of combination therapy with rosiglitazone and losartan brought no greater benefit than rosiglitazone alone according to histopathology. Considering these results, it is acceptable because $50 \mathrm{mg}$ of losartan is less effective than $20 \mathrm{mg}$ of olmesartan. Moreover, it is not thought that higher doses of olmesartan is more effective because in our previous study, olmesartan significantly deteriorated serum creatinine after 1 year, especially in liver cirrhosis patients treated with a 40-mg daily dose [21]. On the other hand, telmisartan may be more effective than losartan or olmesartan. In rat models, telmisartan has shown comparable efficacy to pioglitazone in improving hepatic steatosis, necroinflammation, and fibrosis $[22,23]$. This is likely attributable to its pleiotrophic effects on peroxisome proliferator-activated receptor gamma up-regulation [24]. Therefore, the efficacy of the other ARBs or combination therapy of other drugs for NASH is debatable.

It is a limitation that the present study is an open-label study with a small population. However, we established the sample size calculation $(\mathrm{N}=7)$ in advance according to Yokohama et al.'s study in which the significant reduction of TGF-beta1 after 1 year was more than $40 \%$ in the ARB group [8]. Moreover, there had only been one olmesartan translational study for NASH at the time we began our study [11]. Therefore, we performed the present small open-label study first.

In conclusion, 1-year of administration of olmesartan significantly reduced MBP and serum inflammation markers but did not improve fibrotic markers or histological features. Therefore, we concluded that olmesartan is advisable only for its anti-inflammatory effect in patients with NASH complicated with hypertension.

\section{ACKNOWLEDGEMENTS}

The authors thank the pathology department staff of Kitasato University East Hospital for their technical assistance. We also thank Robert E. Brandt (Founder, CEO, and CME of MedEd Japan) for editing the manuscript.

\section{REFERENCES}

[1] Chalasani, N., Younossi, Z., Lavine, J.E., et al. (2012) The diagnosis and management of non-alcoholic fatty liver disease: Practice guideline by the American Gastroenterological Association, American Association for the Study of Liver Diseases, and American College of Gastroenterology. Hepatology, 55, 2005-2023. doi:10.1002/hep. 25762

[2] Ekstedt, M., Franzén, L.E., Mathiesen, U.L., et al. (2006) Long-term follow-up of patients with NAFLD and elevated liver enzymes. Hepatology, 44, 865-873. doi:10.1002/hep. 21327

[3] Yoshiji, H., Kuriyama, S., Yoshii, J., et al. (2001) Angiotensin-II type 1 receptor interaction is a major regulator for liver fibrosis development in rats. Hepatology, 34, 745-750. doi:10.1053/jhep.2001.28231

[4] Gaedeke, J., Peters, H., Noble, N.A. and Border, W.A. (2001) Angiotensin II, TGF-beta and renal fibrosis. Contributions to Nephrology, 135, 153-160. 


\section{doi:10.1159/000060162}

[5] Nagy, P., Scaff, Z. and Lapis K. (1991) Immunohistochemical detection of transforming growth factor-beta 1 in fibrotic liver diseases. Hepatology, 14, 269-273. doi:10.1002/hep.1840140211

[6] Matsuoka, M. and Tsukamoto, H. (1990) Stimulation of hepatic lipocyte collagen production by Kupffer cell-derived transforming growth factor beta: Implication for a pathogenetic role in alcoholic liver fibrogenesis. Hepatology, 11, 599-605. doi:10.1002/hep.1840110412

[7] Nagy, P., Scaff, Z. and Lapis, K. (1991) Immunohistochemical detection of transforming growth factor-beta 1 in fibrotic liver diseases. Hepatology, 14, 269-273. doi:10.1002/hep.1840140211

[8] Yokohama, S., Yoneda, M., Haneda, M., et al. (2004) Therapeutic efficacy of an angiotensin II receptor antagonist in patients with nonalcoholic steatohepatitis. Hepatology, 40, 1222-1225. doi:10.1002/hep.20420

[9] Mizuno, M., Sada, T., Ikeda, M., et al. (1995) Pharmacology of CS-866, a novel nonpeptide angiotensin II receptor antagonist. European Journal of Pharmacology, 285, 181-188. doi:10.1016/0014-2999(95)00401-6

[10] Oparil, S., Williams, D., Chrysant, S.G., Marbury, T.C. and Neutel, J. (2001) Comparative efficacy of olmesartan, losartan, valsartan, and irbesartan in the control of essential hypertension. Journal of Clinical Hypertension (Greenwich), 3, 283-291. doi:10.1111/j.1524-6175.2001.01136.x

[11] Hirose, A., Ono, M., Saibara, T., et al. (2007) Angiotensin II type 1 receptor blocker inhibits fibrosis in rat nonalcoholic steatohepatitis. Hepatology, 45, 1375-1381. doi:10.1002/hep. 21638

[12] Kleiner, D.E., Brunt, E.M., Van Natta, M., et al. (2005) Design and validation of a histological scoring system for nonalcoholic fatty liver disease. Hepatology, 41, 13131321. doi:10.1002/hep.20701

[13] Brunt, E.M., Janney, C.G., Di Bisceglie, A.M., Neuschwander-Tetri, B.A. and Bacon, B.R. (1999) Nonalcoholic steatohepatitis: A proposal for grading and staging the histological lesions. American Journal of Gastroenterology, 94, 2467-2474. doi:10.1111/j.1572-0241.1999.01377.x

[14] Matthews, D.R., Hosker, J.P., Rudenski, A.S., Naylor, B.A., Treacher, D.F. and Turner, R.C. (1985) Homeostasis model assessment: Insulin resistance and beta-cell function from fasting plasma glucose and insulin concentrations in man. Diabetologia, 28, 412-419. doi:10.1007/BF00280883

[15] Hamaguchi, M., Kojima, T., Takeda, N., et al. (2005) The metabolic syndrome as a predictor of nonalcoholic fatty liver disease. Annals of Internal Medicine, 143, 722-728. doi:10.7326/0003-4819-143-10-200511150-00009

[16] Torres, D.M., Jones, F.J., Shaw, J.C., Williams, C.D., Ward, J.A. and Harrison, S.A. (2011) Rosiglitazone versus rosiglitazone and metformin versus rosiglitazone and losartan in the treatment of nonalcoholic steatohepatitis in humans: A 12-month randomized, prospective, open-label trial. Hepatology, 54, 1631-1639. doi:10.1002/hep. 24558

[17] Sanyal, A.J., Chalasini, N., Kowdley, K.V., et al. (2010) Pioglitazone, vitamin E, or placebo for nonalcoholic steatohepatitis. New England Journal of Medicine, 362, 1675 1685. doi:10.1056/NEJMoa0907929

[18] Aithal, G.P., Thomas, J.A. and Kaye, P.V. (2008) Randomized, placebo-controlled trial of pioglitazone in nondiabetic subjects with nonalcoholic steatohepatitis. Gastroenterology, 135, 1176-1184. doi:10.1053/j.gastro.2008.06.047

[19] Belfort, R., Harrison, S.A., Brown, K., et al. (2006) A placebo-controlled trial of pioglitazone in subjects with nonalcoholic steatohepatitis. New England Journal of Medicine; 355, 2297-2307. doi:10.1056/NEJMoa060326

[20] Ratziu, V., Giral, P., Jacqueminet, S., et al. (2008) Rosiglitazone for nonalcoholic steatohepatitis: One-year results of the randomized placebo-controlled fatty liver improvement with rosiglitazone therapy (FLIRT) trial. Gastroenterology, 135, 100-110. doi:10.1053/j.gastro.2008.03.078

[21] Hidaka, H., Nakazawa, T., Shibuya, A., et al. (2011) Effects of 1-year administration of olmesartan on portal pressure and TGF-beta1 in selected patients with cirrhosis: A randomized controlled trial. Journal of Gastroenterology, 46, 1316-1323. doi:10.1007/s00535-011-0449-Z

[22] Jin, H., Yamamoto, N., Uchida, K., Terai, S. and Sakaida, I. (2007) Telmisartan prevents hepatic fibrosis and enzyme-altered lesions in liver cirrhosis rat induced by a choline-deficient L-amino acid-defined diet. Biochemical and Biophysical Research Communications, 364, 801807. doi:10.1016/j.bbrc.2007.10.083

[23] Fujita, K., Yoneda, M., Wada, K., et al. (2007) Telmisartan, angiotensin II type 1 receptor blocker, controls progress of nonalcoholic steatohepatitis in rats. Digestive Diseases Sciences, 52, 3455-3464. doi:10.1007/s10620-007-9741-4

[24] Yoshida, T., Yamagishi, S., Matsui, T., et al. (2008) Telmisartan, an angiotensin II type 1 receptor blocker, inhibits advanced glycation end-product (AGE)-elicited hepatic insulin resistance via peroxisome proliferator-activated receptor-gamma activation. Journal of International Medical Research, 36, 237-243. doi: $10.1177 / 147323000803600204$ 